\title{
Lattice strain evolution during uniaxial tensile loading of stainless steel
}

\author{
Bjørn Clausen ${ }^{\mathrm{a}, *}$, Torben Lorentzen ${ }^{\mathrm{a}}$, Mark A.M. Bourke ${ }^{\mathrm{b}}$, Mark R. Daymond ${ }^{\mathrm{b}}$ \\ a Materials Research Department, Riso National Laboratory, Roskilde 4000, Denmark \\ ${ }^{\mathrm{b}}$ Manuel Lujan Jr. Neutron Scattering Center, LANSCE, Los Alamos National Laboratory, PO Box 1663, Mail Stop H805, Los Alamos, \\ NM 87545, USA
}

Received 18 February 1998; received in revised form 11 August 1998

\begin{abstract}
Applied and residual lattice strains were determined by neutron diffraction during a tensile test of a weakly textured austenitic stainless steel and were compared to the predictions of a self-consistent polycrystal deformation model. Parallel to the tensile axis the model predictions are generally within the resolution of the diffraction measurements, but perpendicular to the tensile axis discrepancies are noted. Discrepancies between model and measurements were greater for the residual lattice strains than during loading. It is postulated that this is because the model does not predict reverse plasticity during unload. (C) 1999 Elsevier Science S.A. All rights reserved.
\end{abstract}

Keywords: Neutron diffraction; Time-of-flight; Self-consistent; Polycrystal deformation; Elastic-plastic modeling; Austenitic stainless steel

\section{Introduction}

The macroscopic and microscopic deformation of a polycrystal due to an applied load can be modeled using a so-called self-consistent model, coupled with single crystal mechanical properties. However, while the macroscopic predictions can easily be validated, verification at the microscopic level, i.e. whether the stresses and strains in the individual grains are determined correctly, is harder to achieve. Lattice strain predictions of a self-consistent polycrystal deformation model [1,2] have been treated theoretically [3] but here the predictions are compared with experimentally determined lattice strains. Neutron diffraction provides an unique tool for in-situ non-destructive characterization of lattice strains in polycrystalline materials, and has been widely reported, [4-8]. For this study the advantage of using neutrons, rather than conventional X-rays, is the high penetration power, which allows bulk average determination of the $h k l$ specific lattice strains.

\footnotetext{
* Corresponding author. Present address: Manuel Lujan Jr. Neutron Scattering Center, Los Alamos National Laboratory, PO Box 1663, Mail Stop H805, Los Alamos, NM 87545, USA. Tel.: + 1505 6672944; fax: + 1505 6652676; e-mail: clausen@lanl.gov
}

\section{Material characteristics}

The material used in the present work was an austenitic stainless steel alloy with the composition given in Table 1. The material was supplied in the form of a rolled plate, from which tensile specimens were cut with the longitudinal axis parallel to the rolling direction.

The initial texture of the material affects both the macroscopic behavior and the lattice strain development, and in order that adequate comparisons can be made between theory and experiments, it is necessary that the calculations are based on the actual texture of the material. Therefore an experimental characterization of the texture is required, which can also be done using neutron diffraction. By measuring three pole figures [9] it is possible to establish the orientation distribution function (ODF). The ODF of the material in question, presented in Fig. 1, shows a relatively weak cube texture with a maximum level of about two times random.

Analysis of the microstructure of the material is also necessary to confirm the validity of the neutron diffraction results. The average grain size was measured to be $28 \mu \mathrm{m}$, but as seen in Fig. 2 the grain size distribution 
is rather broad. This small grain size ensures that the diffraction experiments allow powder diffraction assumptions, since there is a sufficiently large number of grains in the gauge volume to give acceptable volume averages of the specific lattice strains.

\section{Experimental procedure}

The diffraction measurements were carried out using the Neutron Powder Diffractometer (NPD) at the Lujan Center at Los Alamos National Laboratory using the time-of-flight (TOF) technique. Elastic lattice strains were measured simultaneously for $15 \mathrm{hkl}$ reflections parallel $(\|)$ and perpendicular $(\perp)$ to the tensile axis, using a stress-rig designed for use on the NPD instrument, [10].

The stainless steel sample was loaded in tension to a maximum stress of $330 \mathrm{MPa}$, and in-situ strain measurements were made at the load levels shown by the symbols in Fig. 3. A nominal load of $5 \mathrm{MPa}$ was used as the zero point to preclude any slight movement of the sample on load or unload (that might result in artificial strain due to the change in scattering geometry). Accordingly the stress-free reference values for each reflection were taken at $5 \mathrm{MPa}$ tensile load. By doing so any residual strains present prior to loading are ignored. During the load cycle the sample was unloaded at $0.2,0.7,1.2$ and $2.0 \%$ plastic strain (indicated by the dotted lines in Fig. 3) to allow measurement of the evolving residual lattice strains. The test was performed under load control, and some room temperature creep was noted during the necessary holding time to record the diffraction pattern, i.e. at 330 $\mathrm{MPa}$ it crept $0.37 \%$ while creep was negligible at 220 $\mathrm{MPa}$. Stainless steel is a relatively good neutron scatter and the measurement of a complete diffraction pattern was possible in $90 \mathrm{~min}$.

The sample is placed in the stress-rig at a $45^{\circ}$ angle to the incident beam which is defined by the slits, S. The two detector banks (D) at plus and minus $90^{\circ}$ measure the transverse, and longitudinal strain components, respectively. A typical diffraction pattern is shown in Fig. 4(b) where the symbols represent the measured intensity and the line is the fit predicted by the Rietveld least squares refinement $[13,14]$. The lower curve is the difference between the measured and Rietveld predicted intensities.

Table 1

Chemical composition of the stainless steel in weight percent

\begin{tabular}{lllllll}
\hline & $\mathrm{Cr}$ & $\mathrm{Ni}$ & $\mathrm{Mo}$ & $\mathrm{Mn}$ & $\mathrm{Si}$ & $\mathrm{C}$ \\
\hline Stainless steel & 18.25 & 13.42 & 3.66 & 1.48 & 0.44 & 0.02 \\
\hline
\end{tabular}

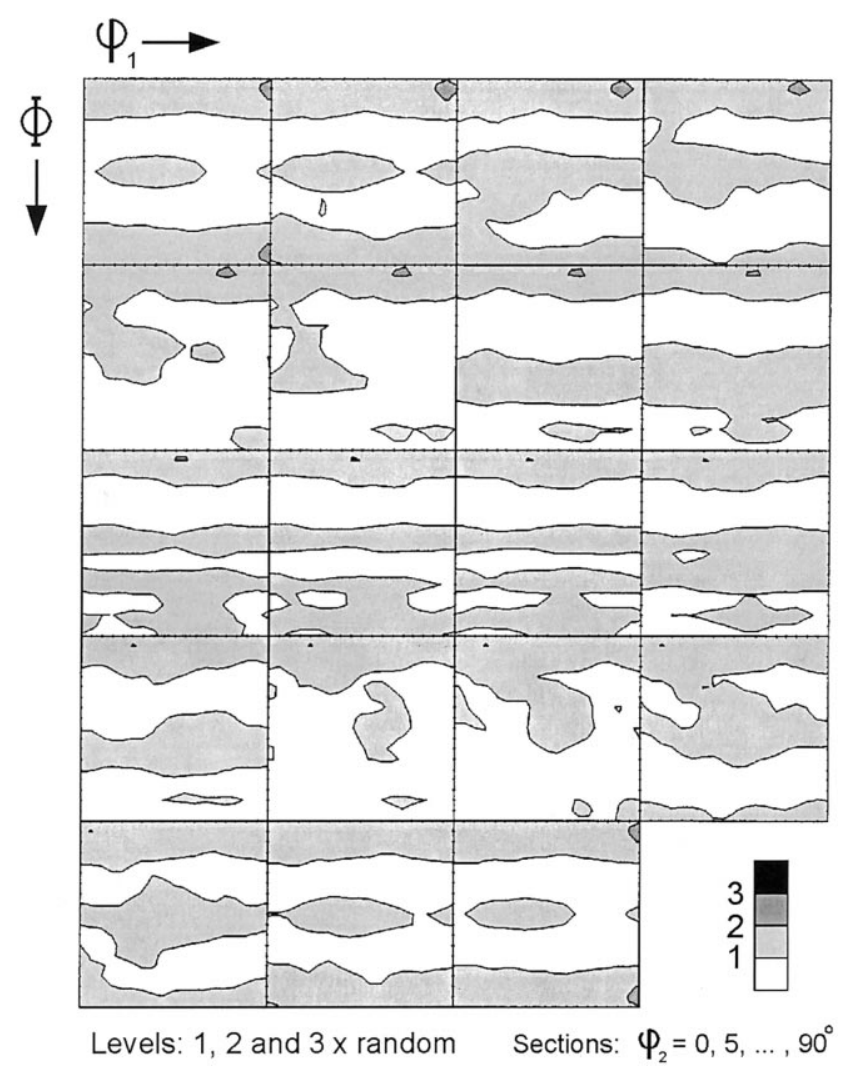

Fig. 1. Orientation distribution function for the stainless steel.

The Rietveld analysis fits the entire diffraction pattern to determine an overall crystal structure with multiple variables including the atom positions and lattice parameters. However, this study addresses the strain development measured using specific $h \mathrm{kl}$ reflections and therefore we used single peak fits, as described in [14], to determine the peak positions and thereby the lattice spacings, $d_{h k l}$. A comparison between Rietveld and single peak fits is made in [15].

In the TOF measurements the full diffraction pattern is recorded simultaneously (from 0.4 to $4 \AA$ ), but the chosen counting time and the overlapping of peaks at low $d$ values (lattice spacing) limited the number of peaks which were measured with acceptable accuracy to about 15 . The smallest $d$ spacing is about $0.544 \AA$ for the 622 reflection. In this work, however, only eight $h k l$ reflections have been compared with the self-consistent model calculations $(111,200,220,311,331,420,422$, 531). Higher order reflections (222, 400 etc.) and reflections corresponding to two families of lattice planes were ignored (511 and 333, 442 and 600). This means that the reflections with Miller indices up to (531), which is the asymmetrical $f c c$ reflection (48 permutations) with the lowest indices, are used. The omitted reflections $(620,533$ and 622) showed similar accuracy to the other peaks, but are not reported for clarity in the illustrations. 


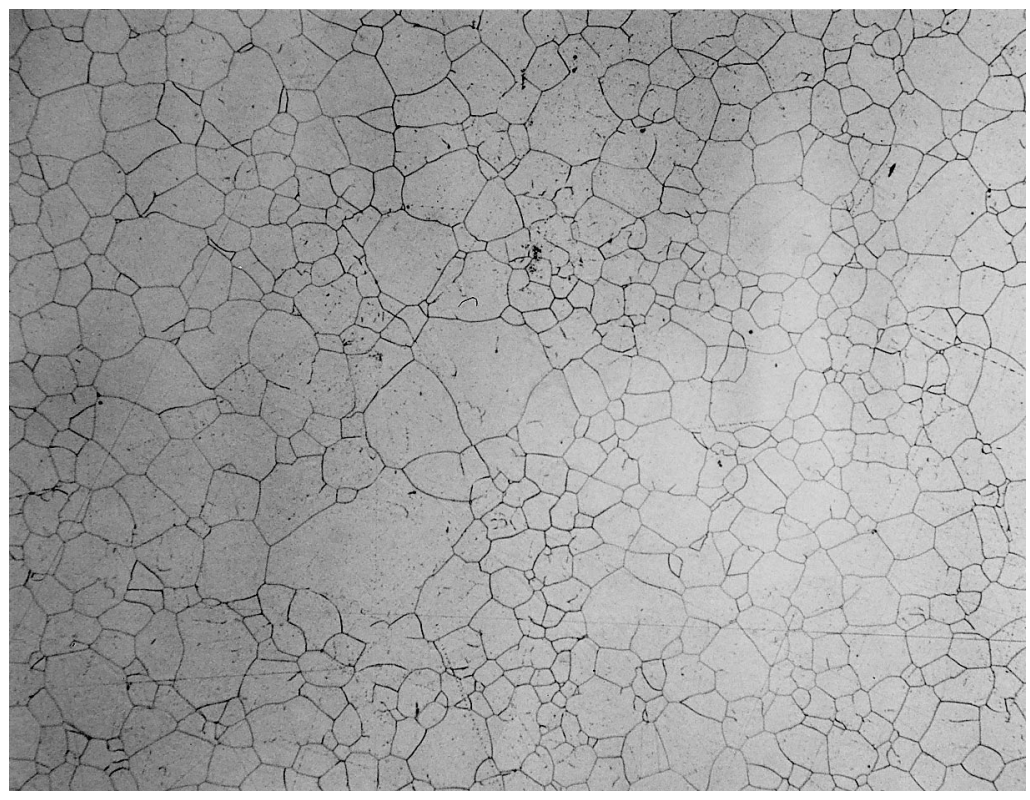

Fig. 2. Micrograph of the stainless steel $(\times 100)$.

The $90^{\circ}$ detectors on NPD sublend a total angle in $2 \theta$ of 84.5 to $95.5^{\circ}$. Accordingly the measured elastic lattice strains are averaged over an angle of $5.5^{\circ}$ within the scattering plane. In order to minimize differences in the comparison between measurements and model predictions we averaged the calculated elastic strain over a similar angular range.

\section{Model}

In the model calculations the initial critical resolved shear stress, $\tau_{0}$, and the hardening parameters, $q, h_{\text {final }}$,

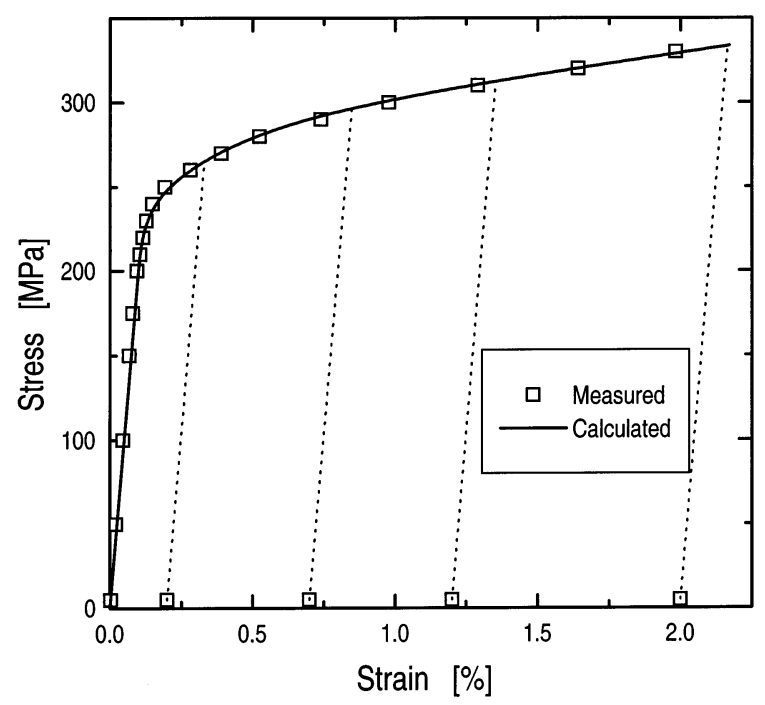

Fig. 3. Measured and calculated macroscopic stress strain curve. The dotted lines indicate unloads for residual lattice strain measurements. $h_{\text {ratio }}$, and $h_{\text {exp }}$, for the exponentially decreasing hardening law described in $[2,3]$, were used as fitting parameters to ensure that the calculated macroscopic stress strain curve resembled the measured polycrystal behavior as closely as possible. As seen in Fig. 3, the exponential decreasing hardening law enables a very accurate representation of the measured macroscopic stress strain curve using the fitting parameters shown in Table 2.

Having ensured an identical macroscopic material response, we proceed to evaluate the model predictions on a microscopic scale; here by correlating the development of lattice strains in the grains.

From the experimentally determined ODF of the stainless steel sample a set of $\approx 5700$ grains is generated following the procedure described by [11]. The single crystal stiffnesses are also needed in the model calculations, though these material parameters are not readily available for all steels. In the present calculations, values were taken from a $\mathrm{FeCrNi}$ alloy, [12], with a slightly different composition $(19 \% \mathrm{Cr}$ and $10 \% \mathrm{Ni})$ than the present stainless steel, see Table 1. This is assumed to have a negligible effect on the outcome of the comparison between theory and experiments.

The single crystal stiffnesses of this $\mathrm{FeCrNi}$ alloy are: $\mathrm{C}_{11}=204.6 \mathrm{GPa}, \mathrm{C}_{12}=137.7 \mathrm{GPa}$ and $\mathrm{C}_{44}=126.2$ $\mathrm{GPa}$, resulting in an anisotropy factor $\left(2 C_{44} /\left(C_{11}+\right.\right.$ $\left.C_{12}\right)$ ) of 3.77. This shows that the austenitic stainless steel has a relatively high degree of elastic anisotropy which is desirable in the present investigations since we are focusing on the interplay between the elastic and plastic anisotropy and the bearing that this has on the evolution of lattice strains. 


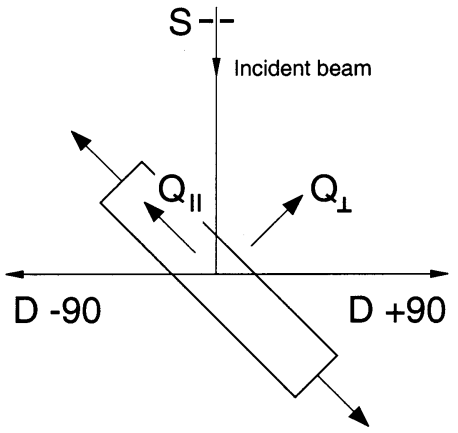

(a) The experimental set-up

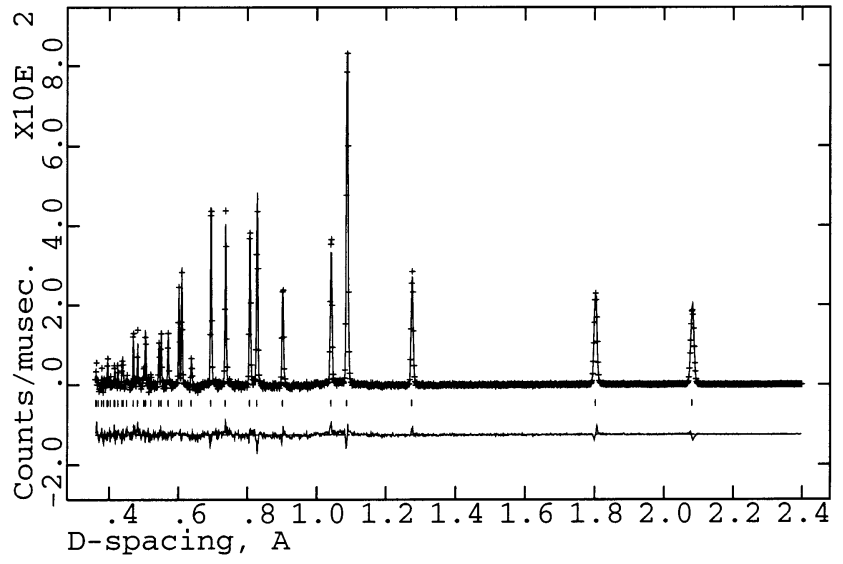

(b) Typical diffraction pattern from the NPD instrument.

for the NPD instrument.

Fig. 4. The experimental set-up and a typical diffraction pattern for the NPD at the Lujan center.

\section{Comparison between model and experiment}

The in-situ lattice strains measured under load, parallel $(\|)$ and perpendicular $(\perp)$ to the tensile axis are presented in Fig. 5. The applied stress is shown as a function of the measured and calculated elastic lattice strains.

The comparison between the model and measurements is simple to make due to the similarities between some of the assumptions in the modeling scheme and the characteristics of the neutron diffraction measurements.

The grains that participate in a neutron diffraction measurement have different neighboring grains and surroundings, and the measured average strain for a reflection is therefore determined for an 'average surrounding' which is very similar to the homogeneous equivalent medium (HEM) assumption used in the Eshelby calculations in the modeling scheme.

\subsection{Elastic regime}

The dotted horizontal lines in Fig. 5 at an applied load of $265 \mathrm{MPa}$ marks the macroscopic $\sigma_{0.2}$ yield limit, but it can be seen that the non-linear behavior of the lattice strain response starts before macroscopic yield for most of the reflections. In practice from Fig. 5 it appears that the 'truly' elastic regime before the reflec-

Table 2

Fitting parameters used in the calculations

\begin{tabular}{llllll}
\hline & $\tau_{0}(\mathrm{MPa})$ & $q$ & $h_{\text {final }}(\mathrm{MPa})$ & $h_{\text {ratio }}$ & $h_{\text {exp }}$ \\
\hline Stainless steel & 87.0 & 1.01 & 300 & 5.0 & 120 \\
\hline
\end{tabular}

tions starts to show deviations from linearity in the applied stress versus elastic lattice strain plot, extends only to about $200 \mathrm{MPa}$. In this truly elastic regime the predicted elastic stiffness of the grain sub-sets in the polycrystalline aggregate are good approximations of the measured diffraction elastic constants (DEC), in both directions $(\|$ and $\perp$ ), as seen from Fig. 5 and Table 3.

The calculated DEC for single orientations and for the grain sub-sets (the reflections shown in Fig. 5) are shown in Table 3. The DEC for the 111 and 200 reflections are less extreme than the single crystal stiffness for these orientations as the grains are each modeled as being embedded in a HEM, [3], and therefore experience the presence of the other grains, resulting in a less extreme response. It should furthermore be noted that the calculated DEC for the reflections are less extreme than the ones calculated for the single orientations. This is due to the averaging over the grains within the sub-sets that represent the reflections. The calculated DEC values for the reflections are all within $\pm 10 \%$ of the measured values.

\subsection{Plastic regime}

Fig. 5 shows that the development of lattice strain is very non-linear once the specimen enters the plastic regime. As grain sub-sets become plastic, they do not accumulate elastic load at the same rate as when they where elastic, causing changes in the partitioning of an incremental load increase between the different grain orientations. In Fig. 5(b) the first measurements that show evidence of the onset of plasticity are for the 531 and 331 reflections which show an upward inflection at about $200 \mathrm{MPa}$. Although it is hard to see in the figure, the model predictions show the same result. 


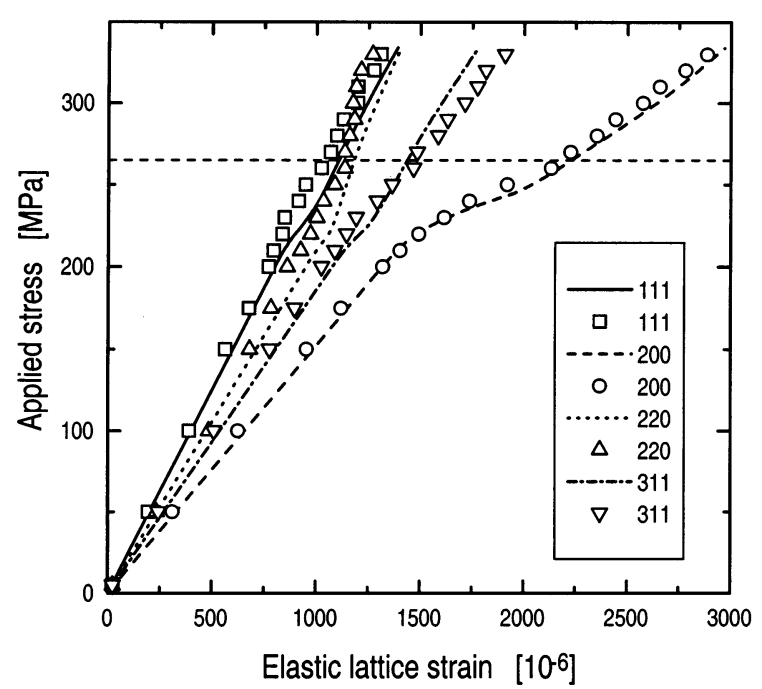

(a) Parallel (\|) to the tensile axis.

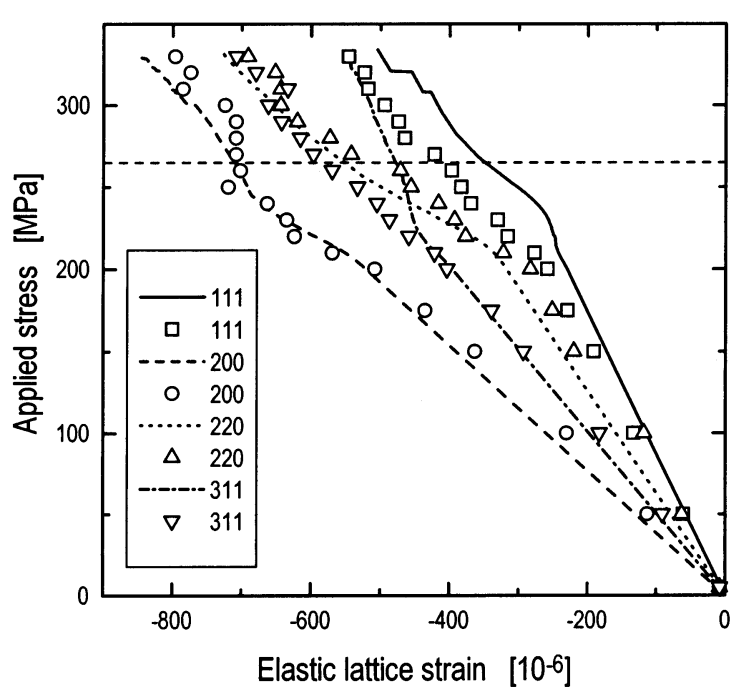

(c) Perpendicular $(\perp)$ to the tensile axis.

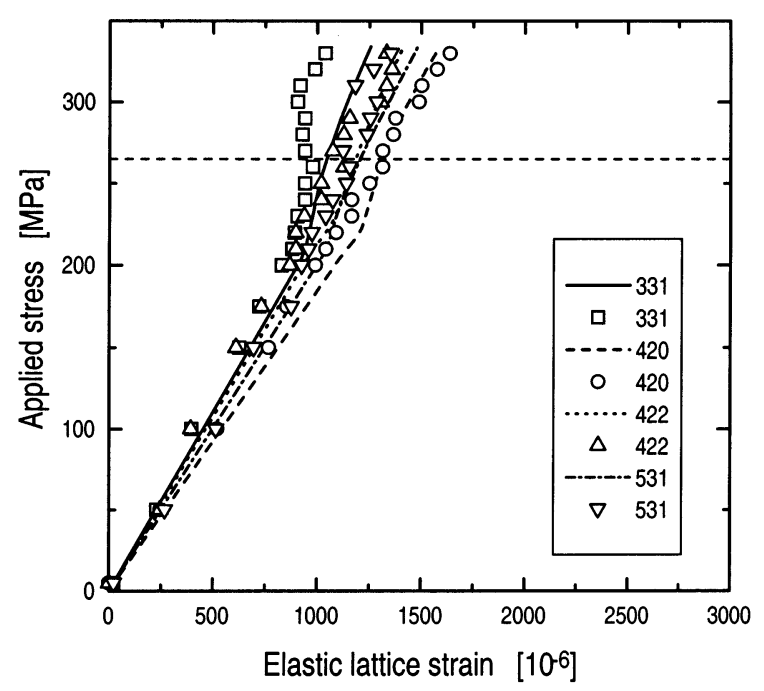

(b) Parallel (\|l) to the tensile axis.

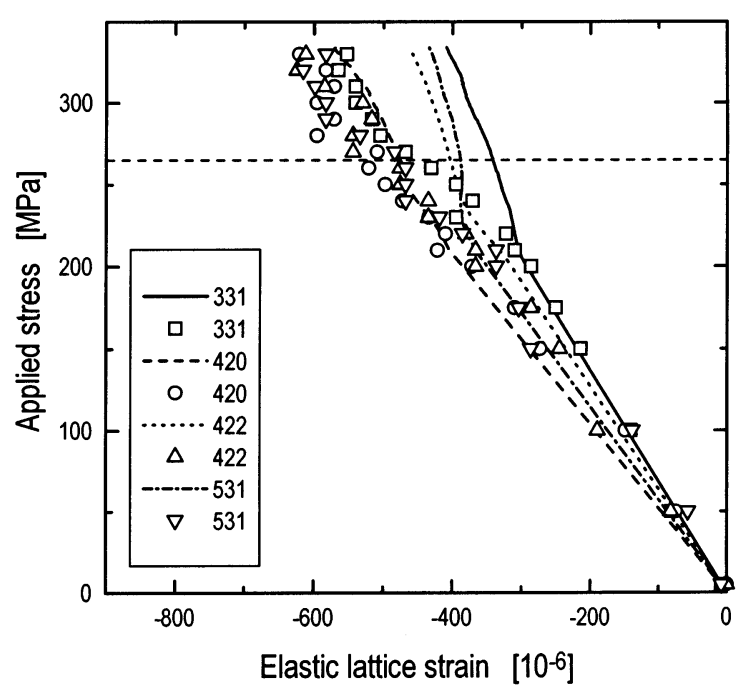

(d) Perpendicular $(\perp)$ to the tensile axis.

Fig. 5. The applied stress versus the elastic lattice strain. Symbols are measuredand lines are calculated. For clarity the data is presented in two graphs for parallel $(\mathrm{a}, \mathrm{b})$ and two graphs for perpendicular $(\mathrm{c}, \mathrm{d})$. The horizontal dotted line represent the macroscopic $0.2 \%$ yield limit.

To examine at which orientations slip is initiated first we present, in Fig. 6, the number of active slip systems in the grains as a function of the grain orientation for two plastic strain levels $(0.001$ and $0.01 \%)$.

Fig. 6(a) is the first step (between 153.5 and 154.9 $\mathrm{MPa}$ ) in the calculation for which some slip systems were activated. Only grains close to the 531 and 331 orientations (indicated by symbols in the figure) have become plastic. When the first grain orientations yield, the remaining orientations, that are still elastic, bear a larger elastic strain to carry the higher load. The 200 reflection stays elastic the longest due to the high elastic anisotropy in stainless steel, as shown in Fig. 6(b). As it is also the softest of the reflections, it shows the largest deviation from linearity (Fig. 5(a)). When all the grains have become plastic, the lattice strain response for the reflections becomes almost linear again, with gradients determined by a combination of the elastic and plastic anisotropy of the material. 
Table 3

Single crystal stiffnesses, calculated diffraction elastic constants for single orientations and $h k l$ reflections, and measured diffraction elastic constants

\begin{tabular}{lcccccccc}
\hline & $E_{111}$ & $E_{200}$ & $E_{220}$ & $E_{311}$ & $E_{331}$ & $E_{420}$ & $E_{422}$ & $E_{531}$ \\
\hline Single crystal stiffnesses & 299.8 & 93.8 & 193.6 & 138.7 & 215.8 & 140.0 & 193.6 & 166.3 \\
Single orientations & 291.5 & 109.7 & 227.0 & 173.8 & 243.4 & 175.0 & 227.4 & 202.2 \\
Reflections & 246.2 & 149.8 & 212.0 & 183.8 & 220.9 & 183.8 & 212.0 & 199.2 \\
Measured & 261.3 & 155.0 & 222.3 & 192.6 & 243.9 & 195.4 & 238.9 & 205.9 \\
\hline
\end{tabular}

Parallel $(\|)$ to the tensile axis, only the predictions for the 331 reflection deviate significantly from the measured lattice strain response. Perpendicular $(\perp)$ to the tensile axis the discrepancies between model calculations and measurements are more pronounced. Some of the reflections (111, 200, 220 and 420), (see Fig. 5(c) and Fig. 5(d)), show the same good agreement with the measurements as for the parallel case, however, the remaining reflections $(311,331,422$ and 531) are predicted to become stiffer in the plastic regime, where as the measurements show that they in fact becomes slightly softer. This illustrates one of the shortcomings of the present model.

\subsection{Residual strain}

The development of residual lattice strain (predicted and measured) at the unloads indicated by the dotted lines in Fig. 3 is presented in Fig. 7. Parallel (\|) to the tensile axis, the development of residual strains for the reflections are predicted well by the model, though the numerical accuracy for some of the reflections is only within $100 \times 10^{-6}$ and the 200 reflection, which experienced the largest deviations from linearity under load, is within $175 \times 10^{-6}$ of the measured levels.
Perpendicular $(\perp)$ to the tensile axis only the residual strain evolution for the 111,200, 220 and 420 reflections is predicted with acceptable accuracy, Fig. 7(c) and Fig. 7(d), as the remaining reflections show considerable error, even in sign. This is caused by the fact that they were predicted to become stiffer in the plastic regime by the model, but actually they became slightly softer as was shown in Fig. 5(c) and Fig. 5(d).

\section{Discussion}

This comparison between neutron diffraction measurements and predictions of a self-consistent model has shown that the model predictions of the lattice strains under load show agreement with experiments to within the measurement accuracy of $150 \times 10^{-6}$. The good agreement between measured and predicted diffraction elastic constants for the reflections (within $2-10 \%$ ), shown in Table 3 , suggest that the single crystal constants used in the calculations were a reasonable choice. The residual lattice strains for both directions $(\|$ and $\perp$ ), generally show poorer agreement between model predictions and measurements than the lattice strains determined under load. The explanation

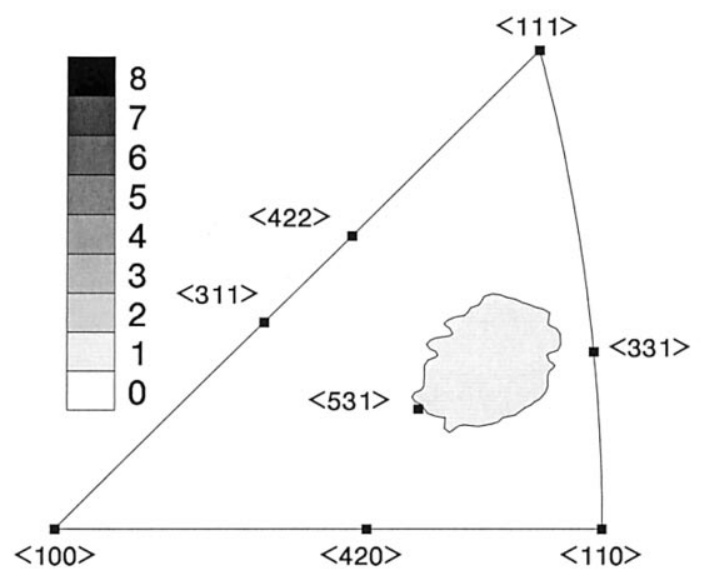

(a) $\varepsilon^{P}=0.001 \%$.

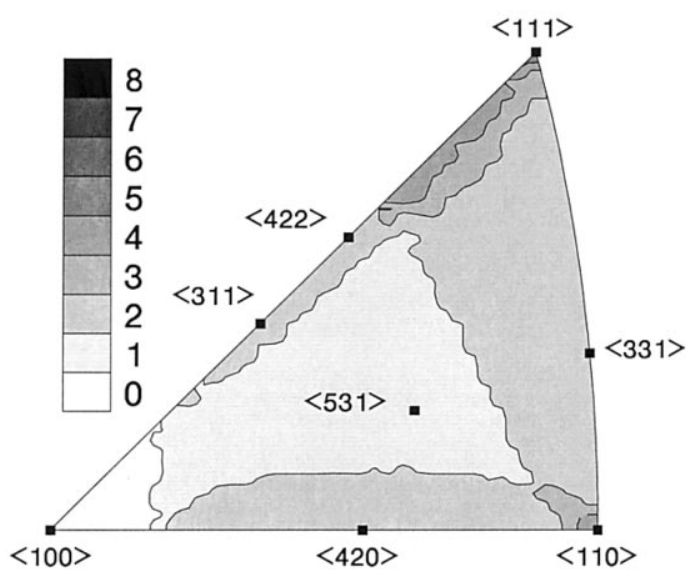

(b) $\varepsilon^{P}=0.01 \%$.

Fig. 6. The number of active slip systems in the grains as a function of the orientation. (a) Plasticity starts close to the 531 and 331 orientations. (b) The 100 orientation is the last to become plastic. 


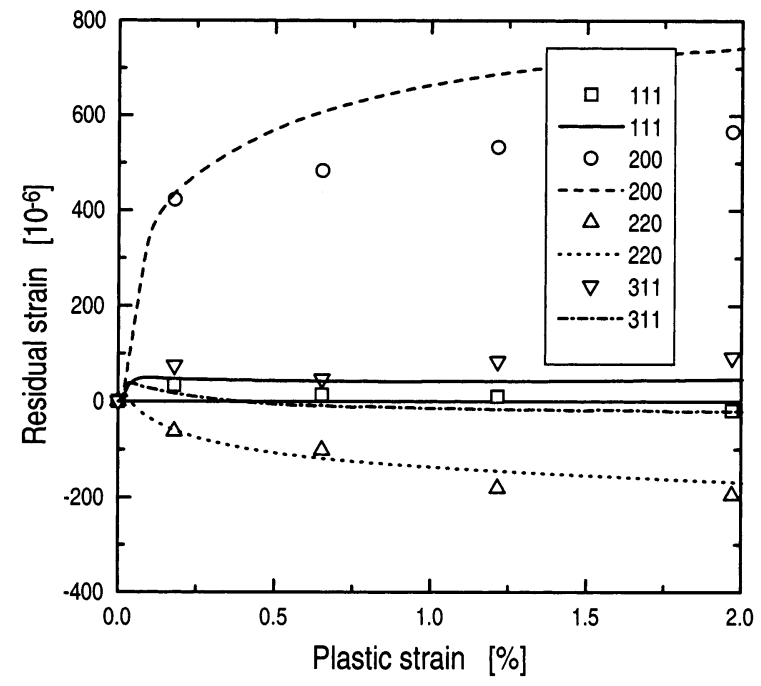

(a) Parallel (II) to the tensile axis.

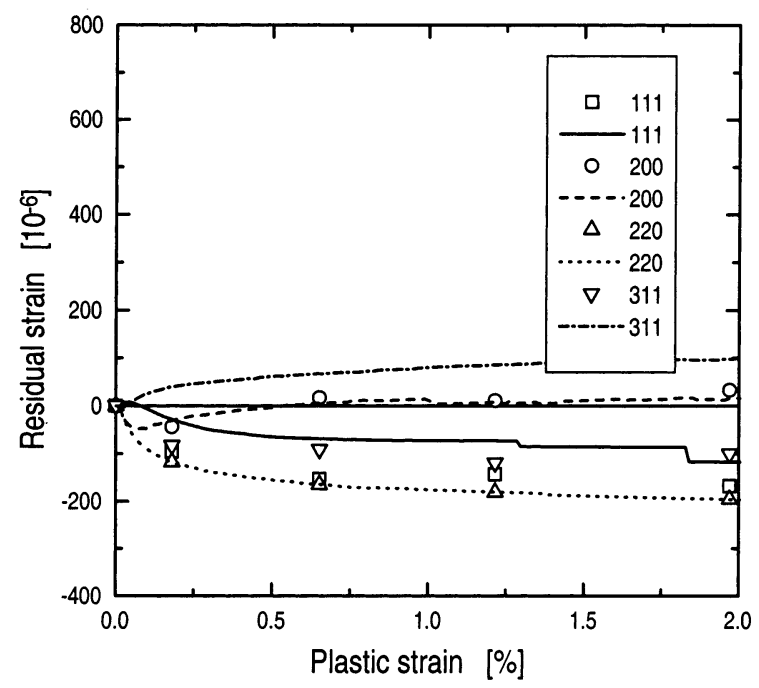

(c) Perpendicular $(\perp)$ to the tensile axis.

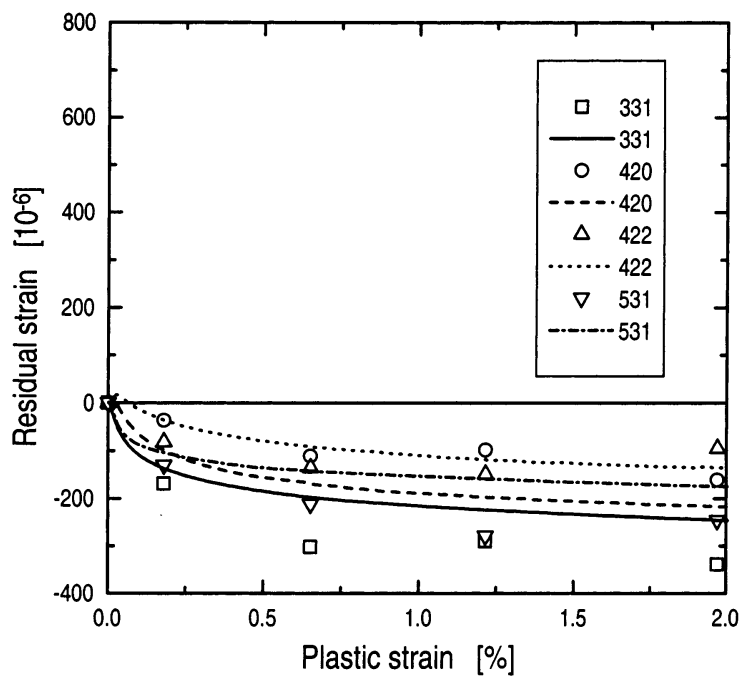

(b) Parallel (II) to the tensile axis.

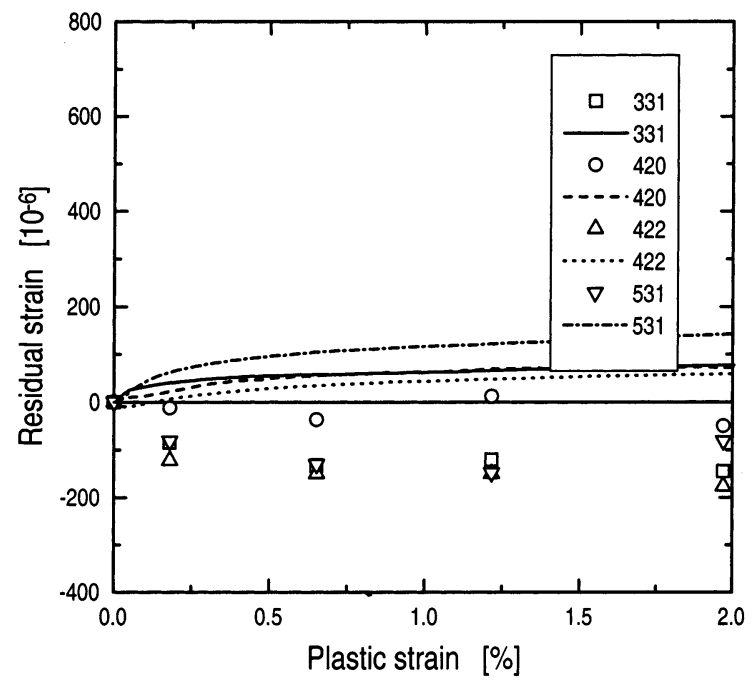

(d) Perpendicular $(\perp)$ to the tensile axis.

Fig. 7. The residual lattice strain as a function of the plastic strain. Symbols aremeasured and lines are calculated.

for the poorer agreement may derive from the fact that the present self-consistent model does not predict slip during unloading. However, the experimental macroscopic measurements show a slight reverse plasticity during the unloads close to zero stress, and some discrepancies between the model predictions and the measurements must therefore be expected. The modeling scheme does predict reverse plasticity when subjected to reverse loading (i.e. compressive), but not for an unload to zero stress.
Another source of uncertainty is the influence of twinning during plastic deformation. The microstructure study revealed that the material does not solely deform by simple crystallographic slip on the well known $f c c$ slip systems $\langle 111\rangle$ (110). Twinning was observed in some grains, though it was by no means the dominant deformation mechanism. An approximation to the effect of twinning has been included in similar self-consistent models, but only for large strain viscoplasticity calculations, [16]. For the low levels of 
plastic strain in the present investigations the volume fraction of twined grains is small $(\approx 10 \%)$ and therefore is expected to have a relatively small effect on the overall deformation.

The weakest link in the modeling scheme is the description of the hardening in the constituents, especially the latent hardening - or the interaction hardening - between slip systems. In the present calculations the hardening matrix, $h_{i j}$, is assumed to be almost isotropic (Taylor hardening). As known from dislocation theory, different junctions between slip systems develop different types of kinks and jogs which again can arrest, or glide undisturbed. This indicates that the matrix should, in fact, be very anisotropic to fully describe the different hardening behavior of the systems.

It is very difficult to measure directly the hardening associated with multiple slip and it is envisaged that comparisons of the type described in this paper can facilitate the investigation of the multislip phenomena in polycrystals. By using different types of hardening laws which do incorporate the dislocation theory (e.g.[17]), and observing the influence on the lattice strain development, it is possible, by following the procedure described in the present work, to investigate the multi slip hardening in polycrystal deformation albeit in a somewhat indirect manner.

At the same time the predictions of the modeling scheme can be very useful in the interpretation of neutron diffraction measurements. In normal engineering measurements the macroscopic stress state is of interest, but the conversion of the measured lattice strains into an overall stress state is not trivial. The influence of the intergranular strains must be considered, and in these cases the modeling scheme can provide the information needed in the calculations.

\section{Conclusion}

The predictions of a one site self-consistent elasticplastic polycrystal deformation model have been evaluated using neutron diffraction measurements of elastic lattice strains in grain sub-sets within a stainless steel polycrystal subjected to uniaxial tensile loading. Comparisons between the measured and calculated lattice strains show that the model, with an isotropic exponential decreasing hardening law, can predict the uniaxial deformation of $f c c$ polycrystals with acceptable accuracy. Predictions of the lattice strain response parallel (\|) to the tensile axis are especially accurate. Perpendic- ular to the tensile axis greater discrepancies are noted. This is partly attributed to the relatively primitive description of the multi slip hardening incorporated in current self-consistent modeling schemes. The generally poorer agreement between predictions and measurements of residual lattice strain is due to the failure to account for reverse yield phenomena on unload.

\section{Acknowledgements}

The theoretical work was carried out within the Engineering Science Centre at Risø National Laboratory in Denmark, while the measurements were carried out at the Manuel Lujan Jr. Neutron Scattering Center at Los Alamos National Laboratory in New Mexico, USA. The Manuel Lujan Jr. Neutron Scattering Center is a national user facility funded by the US Department of Energy, Office of Basic Energy Sciences-Materials Science, under contract number W-7405-ENG-36 with the University of California.

\section{References}

[1] J.W. Hutchinson, Proc. R. Soc. Lond. A319 (1970) 247-272.

[2] B. Clausen, T. Lorentzen, A Self-consistent Model for Polycrystal Deformation, Description and Implementation, Risø-R970(EN), Risø National Laboratory, Roskilde, Denmark, 1997.

[3] B. Clausen, T. Lorentzen, T. Leffers, Acta Mater. 46 (9) (1998) 3078-3089.

[4] A.J. Allen, M.T. Hutchings, C.G. Windsor, C. Andreani, Adv. Phys. 34 (1985) 445-473.

[5] A.J. Allen, M.A.M. Bourke, S. Dawes, M.T. Hutchings, P.J. Withers, Acta Metall. Mater. 40 (9) (1992) 2361-2373.

[6] P.J. Withers, W.M. Stobbs, O.B. Pedersen, Acta Metall. Mater. 37 (1989) 3061-3084.

[7] M.A.M. Bourke, J.A. Goldstone, T.M. Holden, Residual stress measurement using the pulsed neutron source at LANSCE, in: M.T Hutchings, A.D. Krawitz (Eds.), Measurement of Residual and Applied Stress Using Neutron Diffraction. In: NATO ASI Series E, vol. 216, Kluwer, Dordrecht, 1992, pp. 369-382.

[8] M.R. Daymond, P.J. Withers, Appl. Comp. Mater. 4 (6) (1997) 377-394.

[9] D. Juul Jensen, T. Leffers, Text. Microstruct. 10 (1989) 361-373.

[10] M.A.M. Bourke, J.A. Goldstone, N. Shi, J.E. Allison, M.G. Stout, A.C. Lawson, Scripta Metall. Mater. 29 (1993) 771-776.

[11] T. Leffers, D. Junl Jensen, Text. Microstruct. 6 (1986) 231-263.

[12] H.M. Ledbetter, Phys. Stat. Sol. A85 (1984) 89-96.

[13] H.M. Rietveld, J. Appl. Cryst. 2 (1969) 65-71.

[14] R.B. Von Dreele, J.D. Jorgensen, C.G. Windsor, J. Appl. Cryst. 15 (1982) 581-589.

[15] M.R. Daymond, M.A.M. Bourke, R.B. Von Dreele, B. Clausen, T. Lorentzen, J. Appl. Phys. 82 (4) (1997) 1554-1562.

[16] R.A. Lebensohn, C.N. Tome, Mater. Sci. Eng. A175 (1994) 71-82.

[17] J.L. Bassani, Adv. Appl. Mech. 30 (1994) 191-258. 